\title{
Tratamiento médico del "body packer" entre los años 2014 y 2016 en un hospital universitario del caribe colombiano
}

\author{
Medical management of the "body packer" at a university hospital in the \\ colombian caribbean in the period 2014-2016
}

\author{
María Fernanda Mercado", César Rafael Ensuncho², José Carlos Posada³.
}

Médica interna, Facultad de Medicina, Universidad de Cartagena, Cartagena, Colombia.

Médico, residente de Cirugía General, Facultad de Medicina, Universidad de Cartagena, Cartagena, Colombia.

3 Médico, cirujano general; docente, jefe de Sección de Cirugía General, Facultad de Medicina, Universidad de Cartagena, Cartagena, Colombia. Cirujano general, E.S.E Hospital Universitario del Caribe, Cartagena, Colombia.

\section{Resumen}

Introducción. El término "body packer" hace referencia a sujetos portadores de objetos intraabdominales extraños, que contienen drogas ilícitas con fines de contrabando. La mayoría son pacientes asintomáticos, en quienes se instaura conducta expectante, observación clínica estrecha y administración de medicamentos para la evacuación de los paquetes, con miras a prevenir posibles complicaciones, como obstrucción intestinal o intoxicación, asociadas a su transporte intraabdominal.

Materiales y método. Se llevó a cabo un estudio transversal de linealidad retrospectiva en pacientes admitidos en la E.S.E Hospital Universitario del Caribe entre los años 2014 y 20I6,bajo la sospecha diagnóstica de "body packer". Luego de una revisión de las bases de datos institucionales se analizaron variables demográficas y clínicas de los sujetos incluidos en el estudio.

Resultados. Se incluyeron 4 pacientes de género masculino, entre los 22 y 66 años de edad. La cantidad de cápsulas transportadas en promedio fue de 43, para una máxima de 74. La cocaína fue la sustancia que más se identificó. Para la evacuación de los paquetes se empleó irrigación intestinal con polietilenglicol. El tiempo de evacuación máximo fue de 48 horas y no hubo complicaciones asociadas al manejo proporcionado.

Discusión. Estudios respecto al tema, como este, confirman la seguridad del manejo conservador del paciente asintomático y apoyan el uso de polietilenglicol dada su efectividad para lograruna limpieza intestinal completa y por su bajo riesgo de complicaciones asociado a su uso en comparación con otros métodos, así como la menor necesidad de intervenciones quirúrgicas. Se requieren estudios prospectivos aleatorizados controlados a partir de los cuales se determinen, con base en mayor evidencia, las mejores prácticas a seguir.

Palabras clave: transporte intracorporal de contrabando; tráfico de drogas; radiografía abdominal; glicol de etileno.

\begin{abstract}
Introduction. The term "body packer" refers to subjects carrying intraabdominal foreign objects that contain illicit drugs for contraband purposes. The majority of patients are asymptomatic, in whom expectant management is established, with close clinical observation and administration of medications for evacuation of the packages, with prevention of possible complications such as intestinal obstruction or intoxication associated with intraabdominal transport.
\end{abstract}

Fecha de recibido: 30/11/2017 - Fecha aceptación: 02/04/2018

Correspondencia: María Fernanda Mercado Díaz. Cra 6 \# 49 - 65, Chapinero, Bogotá, Teléfono: (57)300-8170499.

Correo electrónico: mafe.mercado95@gmail.com

Citar como: Mercado MF, Ensuncho CR, Posada JC. Tratamiento médico del "body packer" entre los años 2014 y 2016 en un hospital universitario del caribe colombiano. Rev Colomb Cir. 2018;33:135-44. https://doi.org/10.30944/20117582.71 
Materials and method. A retrospective linearity cross sectional study was carried out in patients admitted to the Hospital Universitario del Caribe, Cartagena, Colombia, under the diagnostic suspicion of "body packer" in the period 20I4 and 20I6. After a review of the institutional databases, demographic and clinical variables of the study subjects were analyzed.

Results. Four patients were included, male, ages 22 to 66 years. The average number of capsules transported was 43, with maximum of 74 . Cocaine was the substance mainly identified. Intestinal irrigation with polyethylene glycol was used for intestinal evacuation. The maximum evacuation time was 48 hours and there were no complication associated with the given management.

Discussion. The existing studies on the subject, as well as this one, confirm the safety of the conservative management in the asymptomatic patient and support the effectiveness of polyethyleneglycol in achieving complete intestinal cleansing and the low risk of complications associated with its use with respect to other methods, together with diminished need for surgical intervention. Controlled randomized prospective studies are required to provide greater evidence in order to determine the best practice to be followed.

Key words: body packing; drug trafficking; radiography, abdominal; ethylene glicol.

\section{Introducción}

El término "body packer", conocido coloquialmente como "mula", "culero" o "correo", hace referencia a sujetos portadores de objetos intraabdominales extraños que contienen drogas ilícitas, ingeridos para su ocultamiento con fines de contrabando ${ }^{1}$. No se debe confundir, debido a la diferencia en sus implicaciones clínicas y terapéuticas, con los términos "body stuffer"; sujetos que portan cantidades menores para uso personal, sin fines de contrabando, ingeridas justo antes de ser capturados por las autoridades, y "body pushers", quienes insertan paquetes vía rectal, vaginal e incluso en conducto auditivo externo ${ }^{2}$.

El contrabando por ocultamiento interno se ha convertido en una modalidad bien reconocida para el trasporte de drogas ilegales, constituyéndose en un fenómeno mundial del que se han publicado grandes informes en todos los continentes ${ }^{3,4}$. Aunque es difícil establecer de forma precisa el número de individuos que cruzan las fronteras trasportando cápsulas intraabdominales, se conoce claramente que la región de Centroamérica y el Caribe siguen siendo las principales zonas de procedencia de los "body packer", destacándose históricamente países como Colombia y México, cuyos principales destinos son Estados Unidos, Canadá y diferentes países europeos ${ }^{5,6}$. El embalaje de cuerpos extraños es una práctica peligrosa, que conlleva graves consecuencias médico-legales.
Sin embargo, el desarrollo de mejores métodos de trasporte así como la aparición de nuevas formas de manipulación de estas sustancias, ha permitido evadir las medidas de control sin importar los riesgos que implican el trasporte, el almacenamiento o la distribución de las diferentes drogas recreativas?

El objetivo de este trabajo fue describir el manejo médico del "body packer" en la ciudad de Cartagena entre los años 2014 y 2016 admitidos en la E.S.E Hospital Universitario del Caribe, centro de referencia del departamento de Bolívar.

\section{Materiales y métodos}

Se hizo un estudio de corte transversal, de carácter observacional. Descriptivo, de linealidad retrospectiva, cuya población estuvo constituida por todos los sujetos admitidos con sospecha diagnóstica de "body packer" trasladados a la E.S.E Hospital Universitario de Cartagena, bajo custodia de las autoridades locales, durante el periodo comprendido entre los años 2014 a 20I6, recibidos por el servicio de Cirugía general institucional.

Esta investigación contó con el aval del comité de ética de la E.S.E Hospital Universitario del Caribe.

Se incluyeron pacientes con diagnóstico clínico e imaginológico de "body packer", en tanto que se excluyeron pacientes sospechosos en quienes se descartó el diagnóstico de manera clínica e imaginológica. 
Luego de identificar los "body packers" se revisó la base de datos institucional a partir de lo cual se recolectó información conformada por variables sociodemográficas, modalidad de ingestión y transporte, número de cápsulas evacuadas, sustancia ilícita transportada, tratamiento realizado para evacuación de los paquetes y complicaciones asociadas con el transporte o manejo. Se emplearon medidas de tendencia central para el análisis de los datos cualitativos y cuantitativos.

\section{Resultados}

Se incluyeron 4 pacientes con confirmación diagnóstica de cuerpos extraños en la cavidad intraabdominal, bajo custodia de las autoridades locales, todos de género masculino y nacionalidad colombiana, con un rango de edad entre 22 a 66 años, quienes fueron tratados según el protocolo de "body packer" institucional (figura I). En todos los casos se diagnosticó "body packer" con base en la sospecha clínica-judicial y en la confirmación mediante radiografías simples abdominales sin contraste, que evidenciaban signos radiológicos clásicos ${ }^{8}$ tales como signo del "tictac", "doble condón", "paralelismo" y multiplicidad de paquetes a diferentes niveles del tracto gastrointestinal (tabla I).

Al momento de la admisión todos los pacientes se encontraban asintomáticos, sin hallazgos anormales al examen físico ni alteraciones de sus signos vitales, solo la sospecha epidemiológica y judicial por parte de las autoridades competentes a cargo de la Compañía Antinarcóticos de Control Aeroportuario.

En todos los casos el manejo estuvo enfocado a la evacuación espontánea favorecida con el uso único de agentes para la irrigación intestinal, como polietilenglicol (PEG 3350), a razón de un sobre de polvo diluido en un litro de solución salina $0,9 \%$ e ingestión de cuatro dosis de $250 \mathrm{ml}$ cada 15 minutos, sin necesidad de uso de sonda nasogástrica ni repetición de dosis; o PEG asociado a enema rectal de tipo laxativo salino, sin complicaciones asociadas a su empleo y un tiempo de evacuación promedio de 23 horas (rango de $8-48$ horas).
El número de paquetes expulsados en promedio fue de 43, con un rango de I4 - 74. La cocaína fue la sustancia identificada en la mayoría de los casos; sólo se registró un "body packer" con opio, según el reporte del Cuerpo Técnico de Investigación de la Fiscalía General (CTI). Los pacientes fueron dados de alta médica una vez lograron la deposición de heces libres de paquetes y coincidieron el número de paquetes expulsados con los identificados en la imagen radiológica tomada inicialmente; la tomografía abdomino-pélvica se limitó para confirmar la limpieza completa del tracto gastrointestinal en aquellos casos en los que existía sospecha de permanencia de cápsulas intraabdominales, lo cual no fue necesario en algunos de los casos. Fueron declarados clínicamente limpios y dados de alta médica, y continuaron bajo los procesos judiciales de las autoridades pertinentes.

\section{Discusión}

Como se mencionó anteriormente, el término "body packer" hace referencia a sujetos portadores de objetos extraños intraabdominales que contienen drogas ilícitas, ingeridos para su ocultamiento con fines de contrabando. Deitel y Syed lo usaron por primera vez en 1973 para describir el caso de un hombre de 2I años que presentó un cuadro de obstrucción intestinal tras trece días de haber ingerido un condón con hachís 9 .

La sustancia más trasportada sigue siendo la cocaína en sus diferentes presentaciones (polvo, pasta compacta, líquida), evidenciándose también tráfico de heroína, anfetaminas, marihuana y hachís ${ }^{3,10}$. Cada vez son menos comunes las formas primitivas de empacamiento, las cuales empleaban materiales como condones, dedos de guantes, celofán, plástico, cinta aislante ${ }^{2, I I}$, pues han sido reemplazadas por métodos altamente tecnificados que utilizan varias capas de látex, sellamiento externo en cera e incorporación de papel aluminio o carbón que alteran la radiodensidad y garantizan menor riesgo de fuga o rotura de las cápsulas ${ }^{3}$. Se diferencia de lo que se pensaba anteriormente, no existe un perfil de empacador y aunque la mayoría de los sujetos 


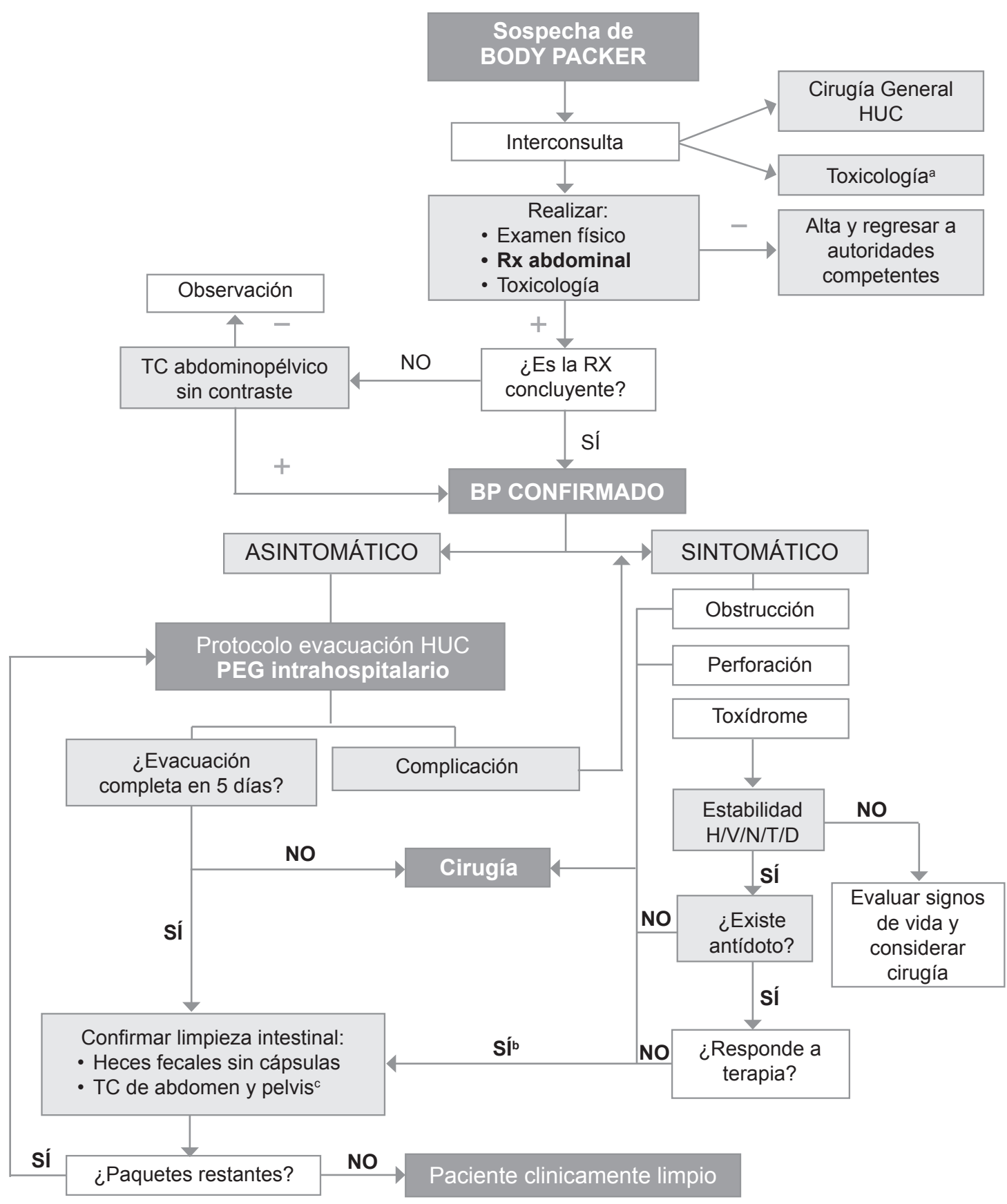

Figura 1. Flujograma para el diagnóstico y manejo de "body packer"s. BP: "body packer"; TC: tomografía computarizada; Rx: radiografía; H/V/N/T/D: hemodinámica/ventilatoria/neurológica/térmica/diurético. Fuente: autores.

aToxicología: seguimiento por grupo de Toxicología institucional para la realización de pruebas de detección de sustancias en muestras biológicas.

bSi responde a terapia con antídoto, continúa con irrigación intestinal. Se debe considerar la necesidad de cirugía según estabilidad clínica y hemodinámica.

cTC de abdomen y pelvis es el método de elección sobre radiografía convencional para confirmación de limpieza intestinal, especialmente cuando se sospecha la permanencia de cápsulas al no concordar el número expulsado con el detectado en la imagen inicial o en los casos donde no se conoce con certeza el número de cápsulas ingeridas. En caso de no disponibilidad, usar como primera línea radiografía de abdomen no contrastada para confirmar evacuación. 
Tabla 1. Variables demográficas y clínicas. Fuente: historias clínicas.

\begin{tabular}{|c|c|c|c|c|c|c|c|c|}
\hline Paciente & Edad & Sexo & $\begin{array}{l}\text { Modalidad } \\
\text { de ingestión }\end{array}$ & $\begin{array}{l}\text { Manejo para } \\
\text { evacuación }\end{array}$ & $\begin{array}{l}\text { Tiempo de } \\
\text { evacuación }\end{array}$ & $\begin{array}{c}\text { Cantidad de } \\
\text { cápsulas } \\
\text { trasportadas }\end{array}$ & $\begin{array}{c}\text { Tipo de } \\
\text { estupefaciente }\end{array}$ & $\begin{array}{c}\text { Complicaciones } \\
\text { asociadas al } \\
\text { trasporte y/o manejo }\end{array}$ \\
\hline 1 & 41 & $M$ & Ingerido & $\begin{array}{c}\text { Enema rectal + } \\
\text { PEG }\end{array}$ & 8 horas & 15 & Opio & Ninguna \\
\hline 2 & 61 & $M$ & Ingerido & $\begin{array}{c}\text { Enema rectal + } \\
\text { PEG }\end{array}$ & 20 horas & 50 & Cocaína & Ninguna \\
\hline 3 & 66 & $M$ & Ingerido & $\begin{array}{l}\text { Estimulación } \\
\text { manual *+ } \\
\text { PEG }\end{array}$ & 12 horas & 35 & Cocaína & Ninguna \\
\hline 4 & 22 & $M$ & Ingerido & $\begin{array}{l}\text { Estimulación } \\
\text { manual *+ } \\
\text { PEG }\end{array}$ & 48 horas & 74 & Cocaína & Ninguna \\
\hline
\end{tabular}

"Estimulación manual: estimulación de la ampolla rectal para favorecer la evacuación espontánea sin que la finalidad sea la extracción manual de cápsulas.

suelen ser hombres, como en este caso, los reportes evidencian la participación de individuos de ambos sexos y de todos los grupos etarios, así como de niños y embarazadas $s^{12,13}$. Aunque el promedio de cápsulas trasportadas en este trabajo fue 43, siendo la cantidad máxima 74, la literatura internacional determina que un "body packer" en promedio trasporta entre 50 y IOO cápsulas, cada una con un contenido de 8 a Io $\mathrm{mg}$ para un total de hasta un I kg de droga aproximadamente; esta cantidad es considerablemente superior en algunos reportes ${ }^{14}$.

De acuerdo con los hallazgos de este trabajo, la mayoría de los "body packers", entre un 80 a $88 \%$, son pacientes asintomáticos, llevados a los centros de salud en custodia de las autoridades, con quienes se sigue una conducta expectante; el porcentaje restante se engloba bajo el concepto de síndrome de "body packer" conformado por todos los individuos que presenten un cuadro de oclusión intestinal o perforación y/o intoxicación por la rotura de una cápsula, secundarios al transporte de paquetes de droga en el tracto digestivo, cuadro clínico que afecta entre el I al 9 $\%$ de los "body packers". La distinción de estos dos escenarios determinará el manejo del paciente, bien sea conservador o quirúrgico 3,15.

El manejo de los que constituyen la mayoría de los "body packers", pacientes asintomáticos, actualmente se enfoca en la evacuación espontánea de los paquetes durante la monitorización y observación continua de signos vitales, previniendo las posibles complicaciones, dado por el enfoque interdisciplinario del servicio de Urgenciología, Toxicología y Cirugía general ${ }^{16}$. Aunque la cirugía temprana alguna vez fue recomendada en este tipo de pacientes, probablemente debido a la alta tasa de ruptura espontánea de los paquetes con envoltura primitiva ${ }^{17}$, con la realización de múltiples estudios sobre el manejo conservador desde los años ochenta se ha reportado una tasa de fracaso del manejo médico conservador del alrededor del $5 \%$, y puede ir en descenso en la medida que la producción de los paquetes mejora, como se evidenció en nuestro trabajo, con una tasa de éxito del Ioo \% con el uso de polietilenglicol (PEG) ${ }^{4,18-21}$. Es así como se ha implementado el uso de múltiples agentes para la irrigación intestinal o laxantes, que garantizan un periodo de expulsión medio de todos los paquetes entre I a 6 días ${ }^{15}$.

El uso de polietilenglicol oral para el lavado intestinal completo, descrito desde I990, ha sido utilizado de forma rutinaria en diversos protocolos para la evacuación de los paquetes de droga debido a la alta tasa de éxito y el bajo riesgo de desarrollar obstrucción intestinal o un toxíndrome tras la rotura de paquetes, especialmente de cocaína; esto último se atribuye al relativo alto pH del PEG que incrementa la tasa de conversión espontánea de la cocaína a su metabolito inactivo, benzoilecgonina ${ }^{15,21-26}$. Se recomienda la administración de I-2 litros de PEG por hora en adultos (dosis que normalmente requiere uso de 
sonda nasogástrica a 250-500 $\mathrm{ml}$ cada I5 minutos), hasta 4 litros al día ${ }^{3}$. En nuestra experiencia, la dosis de I litro por hora de PEG ha sido efectiva ya que ha permitido la evacuación de todas las cápsulas, en un lapso de tiempo no superior a 72 horas, de manera segura y sin complicaciones asociadas. Sin embargo, de forma alternativa se emplean otras sustancias como laxantes oleosos tipo parafina, que aunque resultan eficaces para la evacuación, tienen mayor riesgo de ruptura de la envoltura ${ }^{27}$; catárticos salinos, asociados a trastornos hidroelectrolíticos; carbón activado, cuyo uso se vincula más con los casos de body stuffers o cuando hay liberación de la sustancia para evitar su absorción; y fármacos promotilidad, como eritromicina y metoclopramida, cuyo uso ha sido reportado como seguro en el tratamiento de "body packer"; sin embargo, se requieren más estudios que apoyen su implementación en combinación con $\mathrm{PEG}^{3,28}$. Por consiguiente, el uso de PEG sigue siendo el método de evacuación recomendado en primera instancia en el contexto del paciente asintomático. Adicionalmente, se reconoce que el uso de laxantes rectales en dos de los pacientes estudiados, se basó en la premisa equívoca de conseguir una evacuación rápida, acto suspendido en los casos posteriores al contemplar trabajos científicos que evidenciaban claramente los riesgos asociados a su uso. Es por esto que la implementación de cualquier tipo de laxante o enema rectal se excluye del protocolo propuesto.

La cirugía tiene lugar dentro del manejo del paciente asintomático cuando hay retención de paquetes en el tracto gastrointestinal luego de $5 \mathrm{a}$ 7 días de irrigación, tiempo en el cual existen mayores posibilidades de pérdida de la integridad de las envolturas o fugas de los paquetes, o cuando se expulsan paquetes rotos durante el manejo conservador. De igual manera se considera el manejo quirúrgico en casos de complicación tipo obstrucción, perforación o signo de toxicidad sistémica, en especial cuando se trata de paquetes con cocaína, durante el proceso de evacuación ${ }^{4,8}$. En este contexto también se ha postulado el uso de la endoscopia específicamente en los pacientes asintomáticos en quienes la irrigación intestinal ha fallado y persiste un número pequeño de paquetes que no sobrepasan el píloro ${ }^{29,30}$.

En todos los casos en los que se emplea un manejo conservador y se cree que han sido evacuados completamente los paquetes, están indicados los estudios de imagen para confirmar la descontaminación gastrointestinal total, preferiblemente la tomografía computarizada abdominopélvica sin contraste, que tiene mayor sensibilidad (entre 95,6 - I00 \%) para la detección de un número menor de paquetes en comparación con la radiografía simple de abdomen cuya sensibilidad es menor (entre $85-90 \%$ ) y puede pasar por alto pequeñas cantidades de cápsulas restantes ${ }^{8,31,32}$. Tradicionalmente se ha usado la visualización de 2 a 3 heces libres de paquetes como referencia para confirmar la limpieza gastrointestinal luego de la descontaminación, así como también suele utilizarse el recuento de paquetes, lo cual resulta menos confiable 3 .

Pese a que el "body packer" es un fenómeno bien conocido en Colombia, nunca dejan de existir desafíos al momento de enfrentar cada caso, de ahí que se recomiende la implementación de un enfoque de tratamiento estructurado para garantizar el diagnóstico temprano, la evacuación segura y rápida de los paquetes, y la prevención de las complicaciones. Los trabajos respecto al tema, confirman la seguridad del enfoque conservador, mientras que la intervención quirúrgica se ha convertido en un evento muy infrecuente. Pese a ello, no hay estudios prospectivos aleatorizados controlados a partir de los cuales se determine con mayor evidencia cuáles son las mejores prácticas a seguir. Después de revisar la literatura actual y nuestra propia experiencia, proponemos un protocolo de tratamiento en el que apoyamos el uso de polietilenglicol dada su eficacia y seguridad en el manejo conservador del paciente asintomático.

\section{Conflictos de interés}

Ninguno. 


\section{Referencias}

I. Kulkarni VM, Gandhi JA, Gupta RA, Deokar RB, Karnik ND, Nadkar MY. Body packer syndrome. J Postgrad Med. 2012;58:225-6. doi: I0.4103/0022-3859.I01646.

2. Pidoto RR, Agliata AM, Bertolini R, Mainini A, Rossi G, Giani G. A new method of packaging cocaine for international traffic and implications for the management of cocaine "body packer"s. J Emerg Med. 2002;23:I49-53.

3. Traub SJ, Hoffman RS, Nelson LS. Body packing - The internal concealment of illicit drugs. N Engl J Med. 2003;349:2519-26. doi: I0.I056/NEJMrao22719.

4. Mandava N, Chang RS, Wang JH, Bertocchi M, Yrad J, Allamaneni S, et al. Establishment of a definitive protocol for the diagnosis and management of "body packer"s (drug mules). Emerg Med. J 20II;28:98-IOI. doi: I0.II36/emj.2008.059717.

5. Oficina de las Naciones Unidas contra la Droga y el Delito, Informe mundial sobre las drogas 20I7: resumen, conclusiones y consecuencias en materia de políticas. Fascículo I, 2017. Viena, Austria: Naciones Unidas. 2017.

6. Junta Internacional de Fiscalización de Estupefacientes, Informe de la Junta Internacional de Fiscalización de Estupefacientes correspondiente a 20I6. Nueva York: Naciones Unidas; 2017.

7. Baquero MA. Investigación forense en un caso de "body packer”. Colomb. Forense 2015;2:95-9. doi: I0.I6925/ cf.v3iI.II75.

8. Pinto A, Reginelli A, Pinto F, Sica G, Scaglione M, Berger FH, et al. Radiological and practical aspects of body packing. Br J Radiol. 20I4;87:I-8. doi: I0.I259/ bjr.20130500.

9. Deitel M, Syed AK. Intestinal obstruction by an unusual foreign body. Can Med Assoc J. I973;IO9:2II-2.

Io. Mozes O, Guranda L, Portnoy O, Apter S, Konen E, Amitai MM. Radiographic features of intracorporeally smuggled liquid cocaine. Forensic Sci Med Pathol. 2OI4; IO:535-42. doi: IO.IOO7/sI2O24-0I4-9607.

II. McCarron MM, Wood JD. The cocaine "'body packer"' syndrome. Diagnosis and treatment. JAMA. I983;250:I4I7-20.

I2. Cordero DR, Medina C, Helfgott A. Cocaine body packing in pregnancy. Ann Emerg Med. 2006;48:323-5.

I3. Traub SJ, Kohn GL, Hoffman RS, Nelson LS. Pediatric "body packing". Arch Pediatr Adolesc Med. 2003;157:174-7.

I4. Booker RJ, Smith JE, Rodger MP. Packers, pushers and stuffers--managing patients with concealed drugs in UK emergency departments: a clinical and medicolegal review. Emerg Med J. 2009;26:316-20. doi: Io.II36/ emj.2008.057695.

I5. Madrazo S, Silvio-Esteba L, Secanella L, García-Barraza A, Aranda H, Golda T, et al. Body packer: revisión y experiencia en un hospital de referencia. Cir Esp. 2007;82:139-45.

I6. De Prost N, Lefebvre A, Questel F, Roche N, Pourriat JL, Huchon G, et al. Prognosis of cocaine body-packers. Intensive Care Med. 2005:31:955-8.
I7. Suarez CA, Arango A, Lester JL III. Cocaine- condom ingestion: surgical treatment. JAMA.1977;238:139I-2.

I8. Utecht MJ, Stone AF, McCarron MM. Heroin "body packer"s. J Emerg Med. 1993;II:33-40.

19. Caruana DS, Weinbach B, Goerg D, Gardner LB. Cocaine-packet ingestion. Diagnosis, management, and natural history. Ann Intern Med. 1984;I00:73-4.

20. Bakker JK, Nanayakkara PW, Geeraedts LM Jr, de Lange ES, Mackintosh MO, Bonjer HJ. Body packers: a plea for conservative treatment. Langenbecks Arch Surg. 2012;397:125-30. doi: I0.1007/soo423-0II-0846-z.

2I. Alfa-Wali M, Atinga A, Tanham M, Iqbal Q, Meng A, Mohsen Y. Assessment of the management outcomes of "body packer"s. ANZ J Surg. 20I6;86:82I-5. doi: IO.IIII/ ans.I3226.

22. Hoffman RS, Smilkstein MJ, Goldfrank LR. Whole bowel irrigation and the cocaine body-packer: a new approach to a common problem. Am J Emerg Med. 1990;8:523-7.

23. Farmer JW, Chan SB. Whole body irrigation for contraband bodypackers. J Clin Gastroenterol. 2003;37:I47-50.

24. Guganduin SK, Ananda S. Body packers in Mauritius. Rom Leg Med. 2014;22:55-8. doi: I0.1177/036354 6594022004I8.

25. Markovits N, Kumik D, Halkin H, Guranda L, Cohen A, Katz M, et al. "Body Packers" in Israel: A case Series. Israel Med Association J. 2013;15:639-45.

26. Alipourf-Faz A, Shadnia S, Mirhashemi SH, Peyvandi M, Oroei M, Shafagh O, et al. Assessing the epidemiological data and management methods of "body packer"s admitted to a referral center in Iran. Medicina. 2016;95:e3656. doi: I0.I097/MD.0000000000003656.

27. White N, Taylor K, Lyszkowski A, Tullett J, Morris C. Dangers of lubricants used with condoms. Nature. I988;335:19. doi: I0.1038/3350I9ao.

28. Traub SJ, Su M, Hoffman RS, Nelson LS. Use of pharmaceutical promotility agents in the treatment of "body packer"s. Am J Emerg Med. 2003;21:5II-2.

29. Macedo G, Ribeiro T. Esophageal obstruction and endoscopic removal of a cocaine packet. Am J Gastroenterol. 200I;96:I656-7. doi: I0.IIII/j.I572-024I.2001.03829.x.

30. Rousset P, Chaillot PF, Audureau E, Rey-Salmon C, Becour B, Fitton I, et al. Detection of residual packets in cocaine "body packer"s: low accuracy of abdominal radiography - a prospective study. Eur Radiol. 2015;23:2I46-55. doi: I0.1007/so0330-013-2798-x.

3I. Hamid S, Rashid SN, Saini AM. Characteristic imaging features of "body packer"s: a pictorial essay. Jpn J Radiol. 2012;30:386-92. doi: I0.1007/sII604-0I2-0069-4.

32. Esterson YB, PAtel V, Nicastro J, Friedman B. Plain radiography may underestimate the burden of "body packer" ingestion: a case report. Clin Imaging. 20I7;44:57-6o. doi: IO.IOI6/j.clinimag.20I7.04.006. 